\title{
Stereo-Image Matching Using a Speeded Up Robust Feature Algorithm in an Integrated Vision Navigation System
}

\author{
Chun Liu ${ }^{1,2}$, Fagen Zhou ${ }^{1}$, Yiwei $\operatorname{Sun}^{3}$, Kaichang $\mathrm{Di}^{3}$ \\ and Zhaoqin $\mathrm{Liu}^{3}$ \\ ${ }^{1}$ (College of Surveying and Geo-Informatics, Tongji University, Shanghai, China) \\ ${ }^{2}$ (Key Laboratory of Advanced Engineering Surveying of NASMG, Shanghai, China) \\ ${ }^{3}$ (Institute of Remote Sensing Application, Chinese Academy of Science, \\ Beijing, China) \\ (E-mail: liuchun@tongji.edu.cn)
}

\begin{abstract}
Visual navigation is comparatively advanced without a Global Positioning System (GPS). It obtains environmental information via real-time processing of the data gained through visual sensors. Compared with other methods, visual navigation is a passive method that does not launch light or other radiation applications, thus making it easier to hide. The novel navigation system described in this paper uses stereo-matching combined with Inertial Measurement Units (IMU). This system applies photogrammetric theory and a matching algorithm to identify the matching points of two images of the same scene taken from different views and obtains their 3D coordinates. Integrated with the orientation information output by the IMU, the system reduces model-accumulated errors and improves the point accuracy.
\end{abstract}

\section{KEY WORDS}

1. Vision Navigation. 2. Stereo-matching. 3. Inertial Measurement Units (IMU). 4. Speeded Up Robust Features (SURF).

Submitted: 18 December 2011. Accepted: 23 April 2012. First published online: 12 June 2012.

1. INTRODUCTION. Localization is a key capability of autonomous navigation of vehicles and robots. The Global Positioning System (GPS) has been the most widespread navigational system used in outdoor navigation. However, navigation methods relying on GPS can be vulnerable, and the signals may be disturbed in urban environments with tall buildings or elevated rails. Worse, it is not available in specific areas such as underground locations and outer space. However, integrated vision navigation is a technique that uses single or multiple cameras to acquire $2 \mathrm{D}$ image information from a scene, and then performs the navigation by applying 
algorithms such as image processing, computer vision, and object recognition to locate the 3D dynamic positions. In the non-GPS environment, integrated vision navigation is more advanced than other techniques. It is a passive positioning technique with good imperceptibility, high observation speed, and good accuracy. On the other hand, integrated vision navigation can avoid some strict environmental restrictions because it does not depend on any signal or radiant sources. However, it still becomes a problem when real-time and high-precision performance is required.

DeSouza and Kak (2002) presented an investigation of the developments in the fields related to vision for mobile robot navigation in the past 20 years. The differences in how vision is used for indoor and outdoor robots are large; thus they divided their investigation into two different categories: indoor navigation and outdoor navigation. Indoor navigation was then focused on map-based, map-building-based and mapless navigation. The map-based navigation solution is supported by providing the robot with a model of the environment. It is proposed by providing automated or semiautomated robots that could explore their environment and build an internal representation. However, the mapless navigation solution relies on the integrated navigation system without considering any prior description of the environment. Compared with indoor navigation, outdoor navigation usually involves obstacle-avoidance, landmark detection, map building/updating, and position estimation. Thus, outdoor navigation is concentrated on structured and unstructured environments, and some progress can be efficiently made to represent the uncertainties in a robot's knowledge of its environment as well as its own relative position in the environment.

A first prototype of the vision navigation system 'NavLAB' was developed by Carnegie Mellon University (Thorpe et al., 1988). It mainly considered path tracking and 3D vision. The area correlation-based method is used in path tracking by dividing an image into road and non-road points. This method can adaptively select road models according to different roads and environments, and the most likely road border point is determined based on the acquired road or non-road points to finally achieve path recognition and tracking. After 3D information has been acquired via point-cloud processing from ERIM LIDAR data, 3D vision is performed to optimize the vehicle navigation by recognizing track obstacles and analysing track terrains.

The Chinese Academy of Sciences (Su and Zhu, 2005) presents a design method for a novel configured stereo vision navigation system for mobile robots, which is a catadioptric sensor called the Omnidirectional Stereo Vision Optical Device (OSVOD), based on a common perspective camera coupled with two hyperbolic mirrors. As the hyperbolic mirrors ensure a Single View Point (SVP), the incident light rays are easily found from the points of the image. The two hyperbolic mirrors in OSVOD share one focus that coincides with the camera centre, which is coaxially and separately aligned. Thus, the geometry of OSVOD naturally ensures matched epipolar lines in the two images of the scene. The separation between the two mirrors provides a large baseline and eventually leads to a precise result. The properties mentioned above make OSVOD especially suitable for omnidirectional stereo vision because depth estimation depends on speed, efficiency and precision. The proposed system can be used by mobile robots for obstacle detection, automatic mapping of environments, and machine vision where fast and real-time calculations are needed.

Actually, the application of Visual Odometry (VO) and IMUs on Mars exploration rovers are famous and successful examples; they enabled the rovers to drive safely 
and more effectively in highly-sloped and sandy terrains. After moving a small amount on a slippery surface, the rovers were often commanded to use camera-based VO to correct its errors in the initial wheel odometry-based estimation when the wheels lost traction on large rocks and steep slopes (Cheng et al., 2005; Maimone et al., 2007).

Another successful application of VO/IMU integration is in the natural environment revealed by Konolige et al., (2007). In this algorithm, an Extended Kalman Filter (EKF) was used for VO and IMU data fusion via loose coupling. It is implemented for three steps:

- Step 1. The EKF formulation starts with motion prediction from VO.

- Step 2. The IMU is used as an inclinometer (absolute roll and pitch) to correct the absolute gravity normal.

- Step 3. The IMU is used as an angular rate sensor (for incremental yaw) to correct relative yaw increments.

By using these techniques, the EKF attains precise localization in rough outdoor terrain. As the author presented, a typical result is less than $0 \cdot 1 \%$ maximum error over a $9 \mathrm{~km}$ trajectory, the IMU used in the system with Gyro bias stability of $1 \mathrm{deg} / \mathrm{h}$.

Later, Bayoud developed a mapping system using vision-aided inertial navigation (Bayoud and Skaloud, 2008). The system employs the method of Simultaneous Localization and Mapping (SLAM) where the only external inputs available to the system at the beginning of the mapping mission are a number of features with known coordinates.

Stereo image-matching is one of the key techniques in integrated vision navigation. It is a process of calculating selected features and building relationships between features to match the image points in different images. Image matching is a process that identifies the relationship between a reference image and the image under investigation. A great number of image-matching algorithms have been proposed in recent decades. These algorithms are classified into two categories: pixel-based and featurebased. Pixel-based algorithms can robustly estimate simple transition motion but may fail when dealing with either images with serious transformation or highly degraded images. Optical flow and pixel correlation are two of the most popular pixel-based methods (Lucas and Kanade, 1981; Castro and Morandi, 1987). Feature-based algorithms have recently been widely developed (Mikolajczyk and Schmid, 2005). They can offer a robust image-matching capability when tackling dramatically changed or degraded images, which are invariant to image transition, scaling, rotation, illumination, and limited ranges of viewpoint changes. Feature-based algorithms can have higher image-matching performance than pixel-based algorithms in terms of reliability and precision.

However, feature-based algorithms are not practical for some real-time applications because of the nature of computational complexity and the huge memory consumption. For a feature-based method, Bay et al., (2006) proposed the Speeded Up Robust Feature (SURF) algorithm based on previous research results by considering the time assumption. SURF uses the integral image to approximate the Gaussian convolution, thereby accelerating the convolution process. However, SURF still displays a weakness in matching robustness and results in addition to wrong matching 
points. Moreover, it is still considered too slow to be adopted for real-time applications even though it has significantly improved the processing speed of feature extraction and generation of the feature descriptors.

Song (2004) then analysed the basic theory of binocular stereo vision and studied the key technique of linear feature stereo matching, which is the strategy of camera calibration, feature abstraction, and matching strategy. He first proposed an improved two-step camera calibration method, which obtains interior and exterior orientation elements and lens distortion parameters. At the same time, the proposed method removes control points with large errors and iterations to retrieve more accurate parameters. Song extracted a continuous single-pixel width segment using phase grouping and a heuristic connecting segment extraction algorithm. Finally, he proposed a novel stereo-matching strategy based on the geometry features and corner points of neighbouring segment regions. The matching and calibration results are both optimized with epipolar and parallax continuity constraints.

Results show that although stereo-matching has been developed for years with great achievement, several theoretical and technical problems remain to be addressed. For example, the method based on feature matching produces great computation but is hard to use in high real-time demand applications. The problem of executing a fast match of common points between images is the core issue of stereo-matching. Another challenge in stereo-matching is the ambiguity of the matching results. Under certain circumstances, two independent images share many similar features or pixels, resulting in the resolution of big barriers. The results of stereo-matching can facilitate the fast measurement of the $3 \mathrm{D}$ coordinates of an object; however, a challenge is presented when the image is acquired at high speed, such as in a moving vehicle. Thus, a new vision navigation system based on image matching and photogrammetric theory is proposed in the current paper, and the determination of real-time, $3 \mathrm{D}$ positions from the sequence-based image is also discussed.

2. STEREO CAMERA CALIBRATIONS. One of the most important tasks of computer vision is to reconstruct a $3 \mathrm{D}$ model of the real world and recognize objects by implicitly analysing geometric information included in the images captured by a camera. The spatial geometric information of a point and its corresponding point in the image space is determined by the model parameters of the camera, and in most conditions, these parameters are not easy to know directly. However, they can still be obtained via experiments, known as camera calibration. Camera calibration determines the geometric and optical parameters of the camera, and the orientation and position parameters of the camera coordinate system relative to the object coordinate. This process greatly affects the accuracy of computer vision.

Furthermore, camera calibration determines camera position and property parameters, and establishes the imaging model, which determines the show points in the space coordinate project and their correspondence in the image plane. An ideal optical projection imaging model is the central projection, also known as the pinhole model. The pinhole model assumes that the reflected light is projected onto the image plane, which satisfies the conditions for direct light transmission. The pinhole projection model consists of the centre of the projection, the image plane, and the optical axis (Figure 1). 


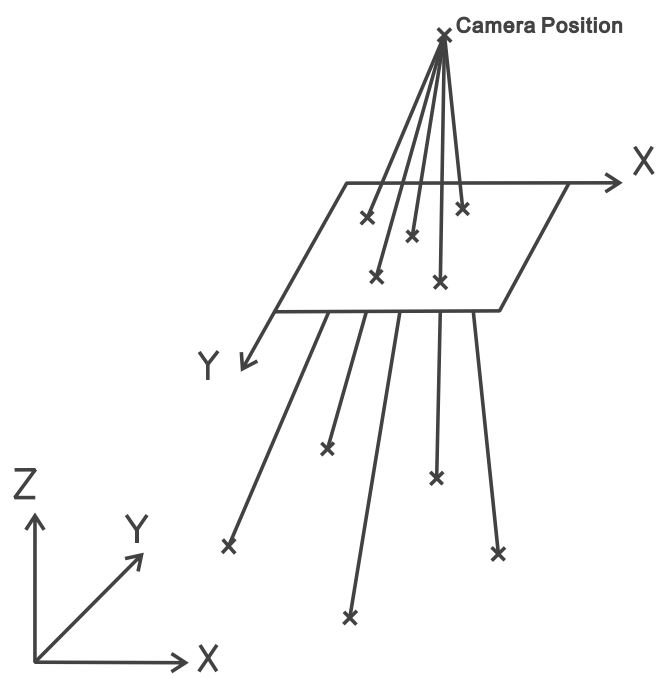

Figure 1. Pinhole projection model.

Most existing camera models are parametric and currently use the following camera calibration methods (Pei, 2010):

- Direct linear transformation method: fewer parameters involved, convenient to compute.

- Perspective transformation matrix method: builds the camera's imaging model through the perspective transformation matrix.

- Two-step camera calibration: initially uses the perspective transformation matrix method to solve the linear camera parameters, and then uses these parameters as initial values, considering the distortion factors to obtain nonlinear solutions using the optimization method.

- Two-plane calibration method.

The camera calibration module of the commercial software iWitness ${ }^{\circledR}$ used in the current experiment can easily access the internal and external parameters of the camera. Figure 2 shows the calibration results using the actual image shooting in Tongji University, Shanghai, China and the corresponding coordinate data. From the experimental results, the camera centre and focal length measured by a nonmeasurement camera exhibits significant deviation from the actual values, resulting in greater deviation in the subsequent resection experiment. Therefore, the camera must be pre-calibrated before a non-measurement camera is used in the measurements; otherwise, the accuracy of the subsequent results would be reduced.

3. SURF-BASED IMAGE-MATCHING ALGORITHM. The purpose of stereo image matching is to find the correspondence in two or more images of the same scene shot from different perspectives. For any kind of stereo-matching method, the effectiveness of the solution depends on three key areas, namely, choosing the right matching features, looking for their essential properties, and then establishing a stable 


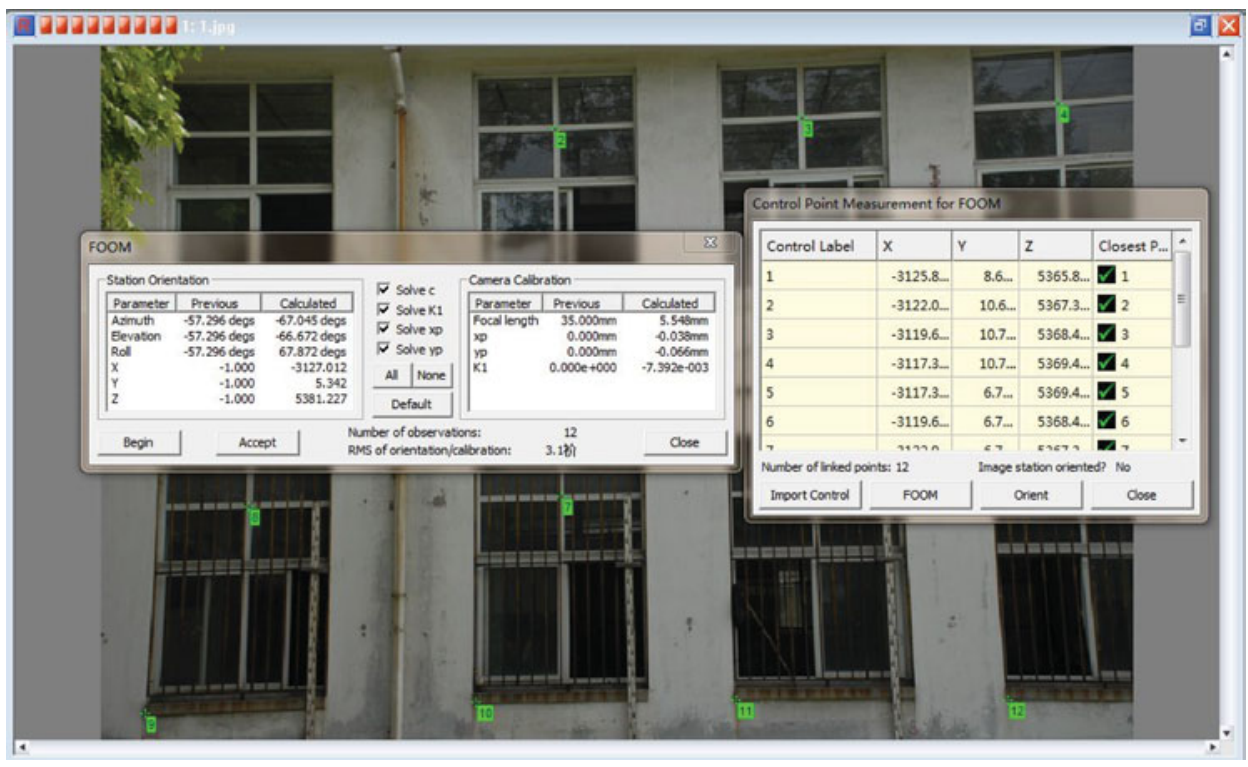

Figure 2. Camera calibration result.

feature-matching algorithm. In the present research, feature selection can be divided into two categories, namely, region-based image correlation matching (area-based matching), and features-based image correlation matching.

According to the selected feature, feature-based matching can be subdivided into point-matching, line-matching and surface-feature matching. In general, featurematching can be done in three steps: (a) feature extraction, (b) feature description, and (c) feature matching. Compared with other matching algorithms, the feature-based matching algorithm does not directly depend on the characteristics of grey, and thus is significantly more robust. Meanwhile, these algorithms can be calculated faster and relatively easily deal with disparity discontinuity regions. However, their matching accuracy largely depends on the accuracy of matching point detection.

3.1. Principle of SURF-Based Image-Matching Algorithm. In 2006, Herbert Bay proposed a novel scale and rotation-invariant interest point detector and descriptor and named it 'Speeded Up Robust Features' (SURF). It approximates and even outperforms previously proposed schemes in the areas of repeatability, distinctiveness, and robustness, and can be computed and compared much faster (Bay, 2006). This performance is achieved by relying on integral images for image convolutions; by building on the strengths of the leading existing detectors and descriptors (specifically the Hessian matrix-based measure for the detector, and a distribution-based descriptor); and by further simplifying these methods.

3.1.1. SURF Algorithm. The SURF algorithm can be divided into three typical steps, namely, accurate interest point localization, interest point descriptor, and feature vector matching (Figure 3).

3.1.2. Matching result of $S U R F$. The program used in the current article is based on the function of the open-source library OPENCV. Figure 4(a) shows the matching result of a standard Graffiti scene, Figure 4(b) shows the result of the surveying 


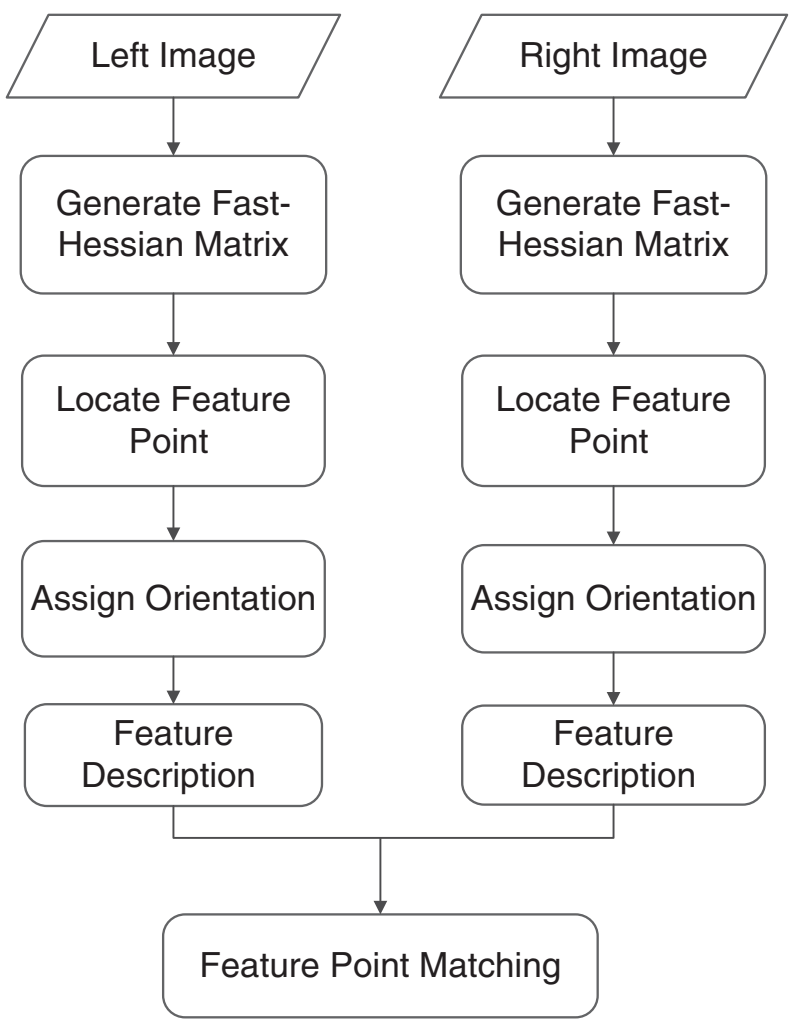

Figure 3. Flow chart of SURF.

building in Tongji University, and Figure 4(c) shows an image of the Germany centre. The size of the three images are $800 \times 640$ pixels, and in each image there are over 200 feature points. These three matching results show remarkable accounts of errormatching points (label 1 is the correct matching point, whereas the labels 2 and 3 are error-matching points). Further improvement is necessary to use these matching results for future measurements.

3.2. Improved SURF-Based Image-Matching Algorithm. SURF significantly improves the matching efficiency. However, it also reduces the matching stability and results in increased error-matching points. Certain measures must be taken to reduce the number of error-matching points because they are highly important in the follow-up application.

3.2.1. Improvement Strategy. Given that most points have achieved correct matching in the original SURF matching process and only a small number obtained error matching, the original matching results are treated as coarse results with gross errors. In addition, because the same $2 \mathrm{D}$ point coordinates in both images have been obtained during the original matching process, the improvement takes the translation, rotation, and scaling parameters between these two images into account using a robust parameter estimation method for estimation, and a certain threshold is set. The deviation between the original and estimated results is compared, and points with low accuracy are removed, thereby obtaining better matching results. The following 


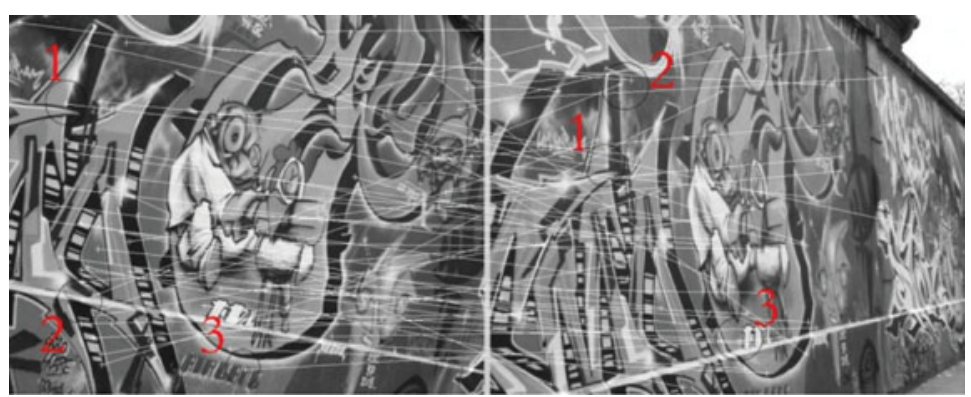

(a) SURF-matching result of a graffiti scene (1/3 points present)

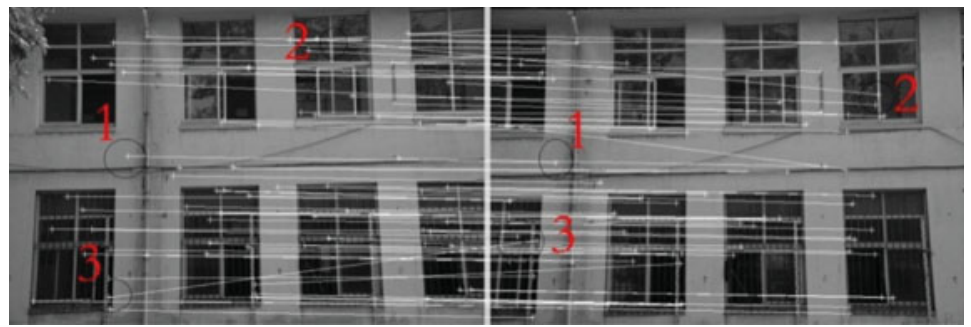

(b) SURF-matching result of a surveying building (1/3 points present)

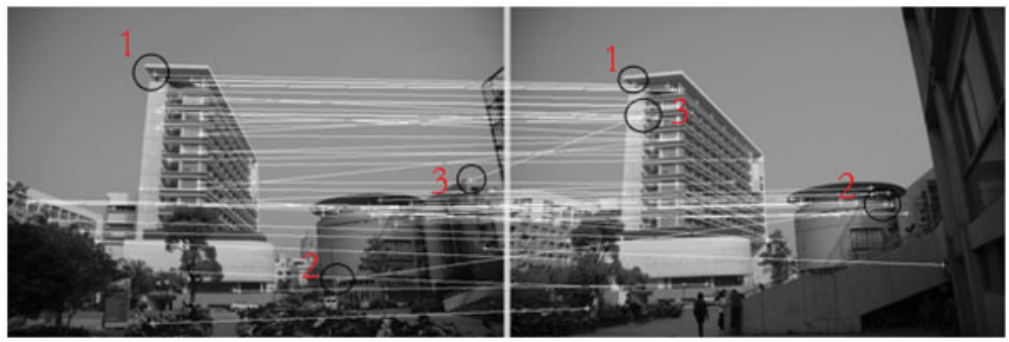

(c) SURF-matching result of the Germany Centre (1/3 points present)

Figure 4. SURF-matching results.

equations show the translation model and the specific robust estimation function used in the current paper.

- Image translation model:

$$
\left(\begin{array}{l}
\mathrm{X} \\
\mathrm{Y}
\end{array}\right)=\left(\begin{array}{l}
\mathrm{X}_{0} \\
\mathrm{Y}_{0}
\end{array}\right)+\mu\left(\begin{array}{cc}
\cos \alpha & -\sin \alpha \\
\sin \alpha & \cos \alpha
\end{array}\right)\left(\begin{array}{l}
\mathrm{A} \\
\mathrm{B}
\end{array}\right)
$$

Assign $c=\mu \cos \alpha, d=\mu \sin \alpha$

$$
\left\{\begin{array}{l}
X=X_{0}+A \times c-B \times d \\
Y=Y_{0}+B \times c+A \times d
\end{array}\right.
$$

- The 'Selecting Weight Iteration' method is used for a robust estimation. The specific method is called a "Norm minimization." Its $\rho$ and weight function are 


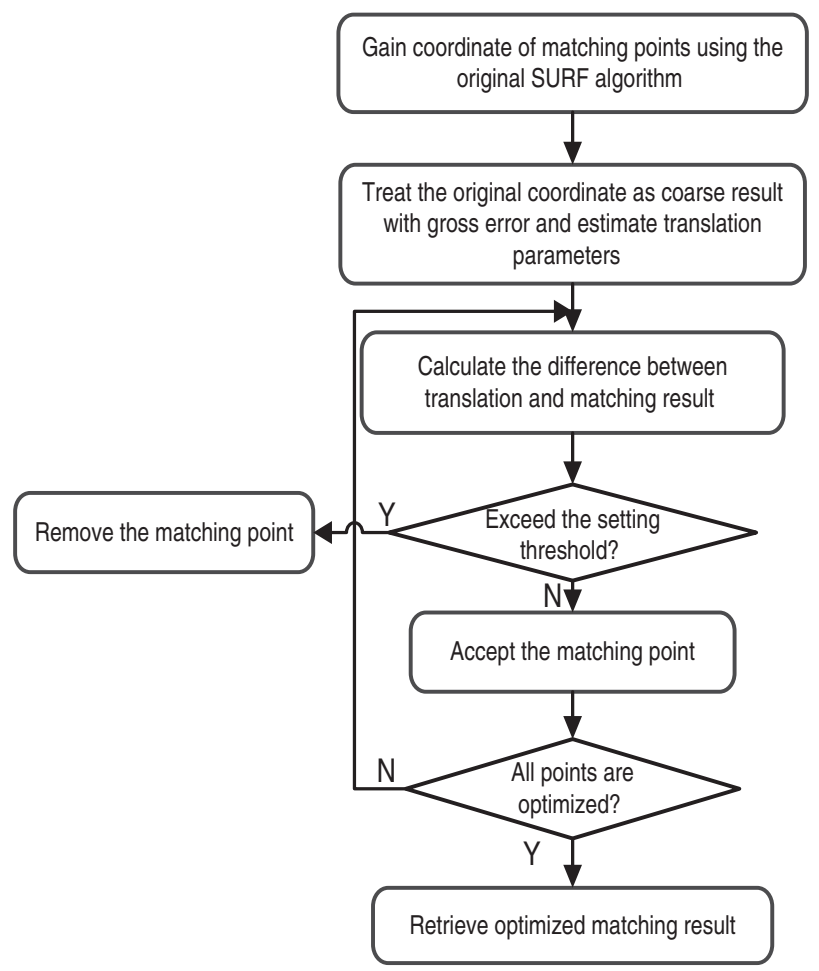

Figure 5. Flow chart of the improved SURF algorithm.

expressed as:

$$
\left\{\begin{array}{l}
\rho=|\mathrm{v}| \\
\mathrm{p}(\mathrm{v})=\frac{1}{|\mathrm{v}|}
\end{array}\right.
$$

The flow chart of the improved SURF algorithm is shown in Figure 5.

3.2.2. Experimental Result of the Improved SURF Algorithm. The same images appearing in the SURF process are used in the current experiment. Figure 6(a) shows the improved result of the standard Graffiti scene and Figure 6(b) shows that of the surveying building. Figure 6(c) shows the Germany centre. The number of matching points in the figures decreases, but the matching accuracy obviously improved. Moreover, the improved SURF algorithm does not significantly increase the execution time (Table 1). Therefore, the improved algorithm appears effective.

\section{THREE-DIMENSIONAL POSITIONING FROM AN IMAGE SEQUENCE.}

4.1. Rough Navigation and Position. In the image sequence, the intersection theory from photogrammetry can be adopted to calculate the corresponding positions of these feature points in the object space if the exterior parameters of the first and second image and the image coordinates of the same point are known. Another resection operation is performed to obtain the orientation and position parameters of the third image. The exterior parameters of the second and third images are already 
Table 1. Comparison between the efficiencies of the original and improved SURF.

\begin{tabular}{lccc}
\hline Picture ID & Image Size & Original $(\mathrm{ms})$ & Improved $(\mathrm{ms})$ \\
\hline 1 & $800 \times 640$ & $9842 \cdot 73$ & $11500 \cdot 9$ \\
2 & $800 \times 640$ & $4054 \cdot 76$ & $5457 \cdot 75$ \\
3 & $800 \times 640$ & $4354 \cdot 27$ & $6317 \cdot 16$ \\
\hline
\end{tabular}

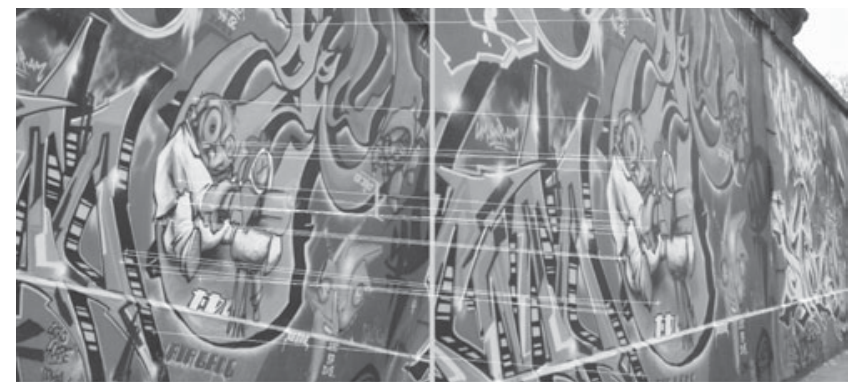

(a) Improved matching of the graffiti scene

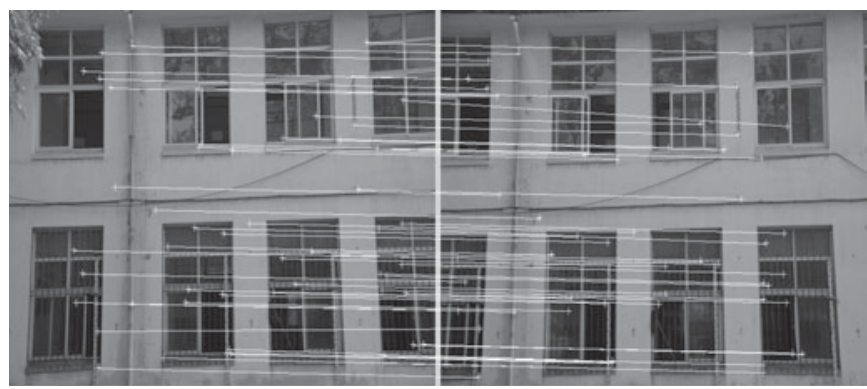

(b) Improved matching of the surveying building (1/3 matching points present)

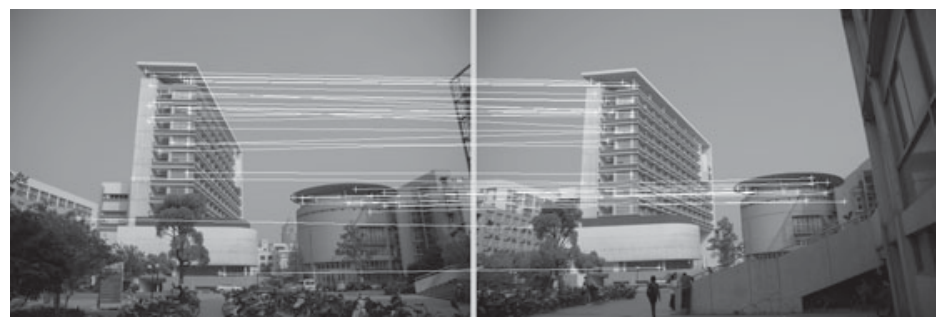

(c) Improved matching of the Germany centre (1/3 matching points present)

Figure 6. Matching results of the improved SURF.

known; hence, the intersection and resection can be continuously operated until a Three Dimensional (3D) coordinate measurement is achieved. The entire process is shown in Figure 7. The specific calculation strategy is as follows:

- The exterior parameters of the first and second images are calculated using the image coordinates of the feature point and the corresponding 3D coordinates in object space (POS1 and POS2 in Figure 7). 


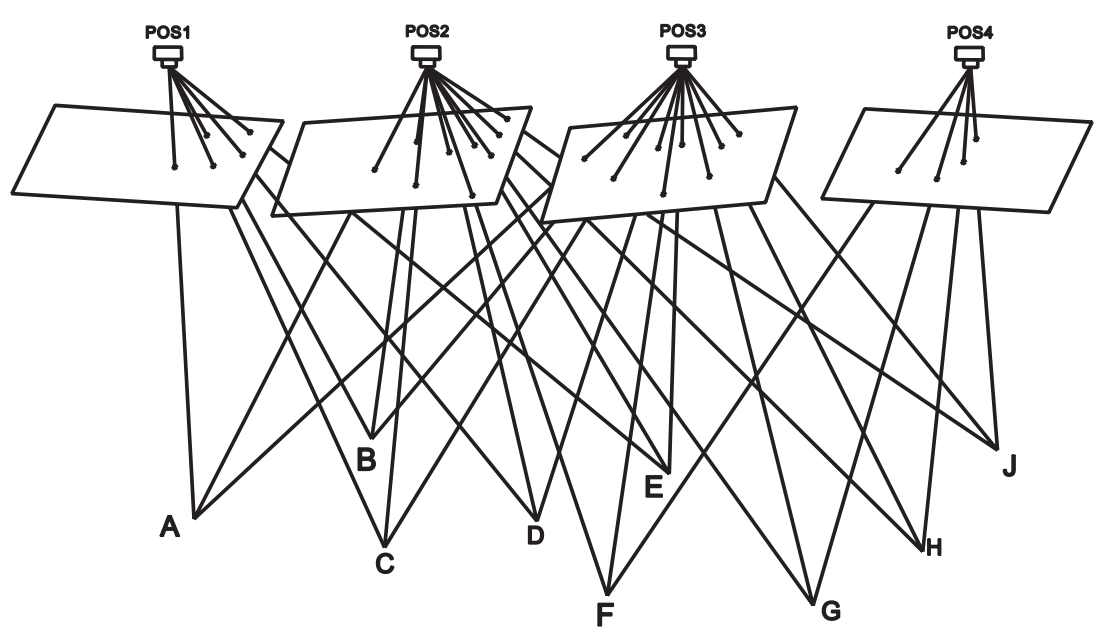

Figure 7. Image sequence-based coordinate measurement.

- The matching points between the first and second image are identified, and the $3 \mathrm{D}$ coordinates of the point in the object space are obtained using the matching result (that is, the coordinates of points $\mathrm{A}, \mathrm{B}, \mathrm{C}, \mathrm{D}$, and E).

- The object coordinates obtained from the second step are passed to the third image using the matching result from the second and third images.

- The exterior parameters of the third image are calculated (obtaining the posture of POS3, as shown in Figure 7).

- The matching points in the second and third images are recalculated and their $3 \mathrm{D}$ coordinates in the object space are obtained using the intersection theory (i.e., the $3 \mathrm{D}$ coordinates of $\mathrm{F}, \mathrm{G}, \mathrm{H}$, and $\mathrm{J}$ are retrieved).

- The same operations from the fourth image are executed until the task is completed.

After finishing the feature matching, the image point coordinates of feature points can be obtained in stereo images based on photogrammetry mathematic model. Here the titled bundle adjustment is used to get the exterior orientation parameters of cameras and the 3D coordinates of unknown ground points as well. Bundle adjustment is based on collinearity equation, making the bundles intersect optimally by means of rotating and transforming. For each matched feature point, two error equations can be formed.

$$
\left.\begin{array}{rl}
v_{x}= & a_{11} d X_{s}+a_{12} d Y_{s}+a_{13} d Z_{s}+a_{14} d \varphi+a_{15} d \omega+a_{16} d \kappa \\
& -a_{11} d X-a_{12} d Y-a_{13} d Z-l_{x} \\
v_{y}= & a_{21} d X_{s}+a_{22} d Y_{s}+a_{23} d Z_{s}+a_{24} d \varphi+a_{25} d \omega+a_{26} d \kappa \\
& -a_{21} d X-a_{22} d Y-a_{23} d Z-l_{y}
\end{array}\right\}
$$

Where, $v_{x}$ and $v_{y}$ are observation residuals of image point coordinates, $a_{11}-a_{16}$, $a_{21}-a_{26}$ are coefficients of error equations, $d X_{s}, d Y_{s}, d Z_{s}, d \varphi, d \omega, d \kappa$ are correction values of exterior orientation parameters, $d X, d Y, d Z$ are correction values of 3D coordinates of unknown ground point, for control points, $d X, d Y, d Z$ are zero, $l_{x}$ and $l_{y}$ are deviation of observation of image point coordinates and approximate 
Table 2. Coordinates on the left image.

\begin{tabular}{|c|c|c|c|c|c|}
\hline \multicolumn{2}{|c|}{$\mathrm{f}=52.5 \mathrm{~mm}$} & \multicolumn{2}{|c|}{$\mathrm{x}=0.0 \mathrm{~mm}$} & \multicolumn{2}{|c|}{$y=0.0 \mathrm{~mm}$} \\
\hline & \multicolumn{3}{|c|}{ Object Space } & \multicolumn{2}{|c|}{ Image Space } \\
\hline & $\mathrm{X}(\mathrm{m})$ & $\mathrm{Y}(\mathrm{m})$ & $\mathrm{Z}(\mathrm{m})$ & $\mathrm{x}(\mathrm{mm})$ & $\mathrm{y}(\mathrm{mm})$ \\
\hline 1 & $5365 \cdot 814$ & $3125 \cdot 820$ & $8 \cdot 655$ & $-29 \cdot 865$ & $4 \cdot 835$ \\
\hline 2 & $5367 \cdot 334$ & $3122 \cdot 043$ & $10 \cdot 698$ & $-4 \cdot 065$ & $17 \cdot 435$ \\
\hline 3 & $5368 \cdot 402$ & $3119 \cdot 686$ & $10 \cdot 704$ & $12 \cdot 035$ & $18 \cdot 135$ \\
\hline 4 & $5369 \cdot 456$ & $3117 \cdot 314$ & $10 \cdot 712$ & $28 \cdot 935$ & $19 \cdot 035$ \\
\hline 5 & $5369 \cdot 468$ & $3117 \cdot 316$ & $6 \cdot 765$ & $30 \cdot 335$ & $-6 \cdot 065$ \\
\hline 6 & $5368 \cdot 400$ & $3119 \cdot 681$ & $6 \cdot 743$ & $12 \cdot 835$ & $-6 \cdot 565$ \\
\hline 7 & $5367 \cdot 348$ & $3122 \cdot 021$ & $6 \cdot 748$ & $-3 \cdot 665$ & $-6 \cdot 765$ \\
\hline 8 & $5366 \cdot 017$ & $3124 \cdot 950$ & $6 \cdot 746$ & $-24 \cdot 165$ & $-7 \cdot 365$ \\
\hline 9 & $5365 \cdot 801$ & $3125 \cdot 802$ & $4 \cdot 715$ & $-31 \cdot 065$ & $-21 \cdot 065$ \\
\hline 10 & $5367 \cdot 116$ & $3122 \cdot 882$ & $4 \cdot 721$ & $-9 \cdot 465$ & $-20 \cdot 265$ \\
\hline 11 & $5368 \cdot 178$ & $3120 \cdot 516$ & $4 \cdot 725$ & $7 \cdot 735$ & $-20 \cdot 065$ \\
\hline 12 & $5369 \cdot 234$ & $3118 \cdot 153$ & $4 \cdot 729$ & $25 \cdot 535$ & $-19 \cdot 965$ \\
\hline
\end{tabular}

Table 3. Coordinates on the right image.

\begin{tabular}{|c|c|c|c|c|c|}
\hline \multicolumn{2}{|c|}{$\mathrm{f}=52.5 \mathrm{~mm}$} & \multicolumn{2}{|c|}{$\mathrm{x}=0.0 \mathrm{~mm}$} & \multicolumn{2}{|c|}{$\mathrm{y}=0.0 \mathrm{~mm}$} \\
\hline & \multicolumn{3}{|c|}{ Object Space } & \multicolumn{2}{|c|}{ Image Space } \\
\hline & $\mathrm{X}(\mathrm{m})$ & $\mathrm{Y}(\mathrm{m})$ & $\mathrm{Z}(\mathrm{m})$ & $\mathrm{x}(\mathrm{mm})$ & $\mathrm{y}(\mathrm{mm})$ \\
\hline 1 & $5366 \cdot 522$ & $3124 \cdot 240$ & 8.670 & $-26 \cdot 265$ & $4 \cdot 135$ \\
\hline 2 & $5367 \cdot 834$ & $3121 \cdot 324$ & $8 \cdot 668$ & -6.065 & $4 \cdot 435$ \\
\hline 3 & $5368 \cdot 899$ & $3118 \cdot 962$ & 8.673 & $10 \cdot 435$ & 4.935 \\
\hline 4 & $5369 \cdot 957$ & $3116 \cdot 598$ & 8.679 & $27 \cdot 735$ & $5 \cdot 435$ \\
\hline 5 & $5369 \cdot 468$ & $3117 \cdot 316$ & $6 \cdot 755$ & $22 \cdot 235$ & $-7 \cdot 365$ \\
\hline 6 & $5368 \cdot 399$ & $3119 \cdot 684$ & $6 \cdot 742$ & $5 \cdot 035$ & $-7 \cdot 565$ \\
\hline 7 & $5367 \cdot 350$ & $3122 \cdot 015$ & $6 \cdot 749$ & $-11 \cdot 465$ & $-7 \cdot 765$ \\
\hline 8 & $5366 \cdot 017$ & $3124 \cdot 948$ & $6 \cdot 745$ & $-32 \cdot 065$ & $-8 \cdot 265$ \\
\hline 9 & $5366 \cdot 506$ & $3124 \cdot 228$ & $4 \cdot 719$ & $-27 \cdot 465$ & $-21 \cdot 665$ \\
\hline 10 & $5367 \cdot 819$ & $3121 \cdot 315$ & $4 \cdot 722$ & $-6 \cdot 165$ & $-21 \cdot 265$ \\
\hline 11 & $5368 \cdot 880$ & $3118 \cdot 947$ & $4 \cdot 730$ & $11 \cdot 235$ & $-21 \cdot 165$ \\
\hline 12 & $5369 \cdot 938$ & $3116 \cdot 582$ & $4 \cdot 728$ & $29 \cdot 635$ & $-21 \cdot 365$ \\
\hline
\end{tabular}

values in the iteration process. For each matched point, an error equation could be established. After all the error equations are established, we would use the iterated solution to get correction values of exterior orientation parameters and 3D coordinates of unknown ground points based on Least-squares principle.

The data shown in Tables 2 to 4 are used in the current experiment. Tables 2 and 3 show the feature point image coordinates and their object coordinates in the first and second images, respectively, which are used to calculate the exterior parameters. Table 4 shows the calculation results, as shown in Figure 8.

4.2. Obtaining the Image Exterior Parameter Through IMU. The relative spatial position and attitude relation between the IMU and the camera must be calculated to facilitate the integration with IMU and achieve optimization (Figure 9). The calibration consists of two steps: (1) determining the offset between the camera 
Table 4. Retrieved posture results.

\begin{tabular}{lrrrrrr}
\hline & $\begin{array}{c}\text { Third } \\
\text { Frame }\end{array}$ & $\begin{array}{c}\text { Fourth } \\
\text { Frame }\end{array}$ & $\begin{array}{c}\text { Fifth } \\
\text { Frame }\end{array}$ & $\begin{array}{c}\text { Sixth } \\
\text { Frame }\end{array}$ & $\begin{array}{c}\text { Seventh } \\
\text { Frame }\end{array}$ & $\begin{array}{r}\text { Eighth } \\
\text { Frame }\end{array}$ \\
\hline $\mathrm{X}(\mathrm{m})$ & $5383 \cdot 453$ & $5384 \cdot 235$ & $5384 \cdot 789$ & $5386 \cdot 316$ & $5385 \cdot 885$ & $5387 \cdot 360$ \\
$\mathrm{Y}(\mathrm{m})$ & $3124 \cdot 235$ & $3122 \cdot 531$ & $3120 \cdot 828$ & $3119 \cdot 173$ & $3117 \cdot 417$ & $3116 \cdot 258$ \\
$\mathrm{Z}(\mathrm{m})$ & $5 \cdot 138$ & $5 \cdot 145$ & $5 \cdot 158$ & $5 \cdot 153$ & $5 \cdot 178$ & $5 \cdot 124$ \\
\hline
\end{tabular}

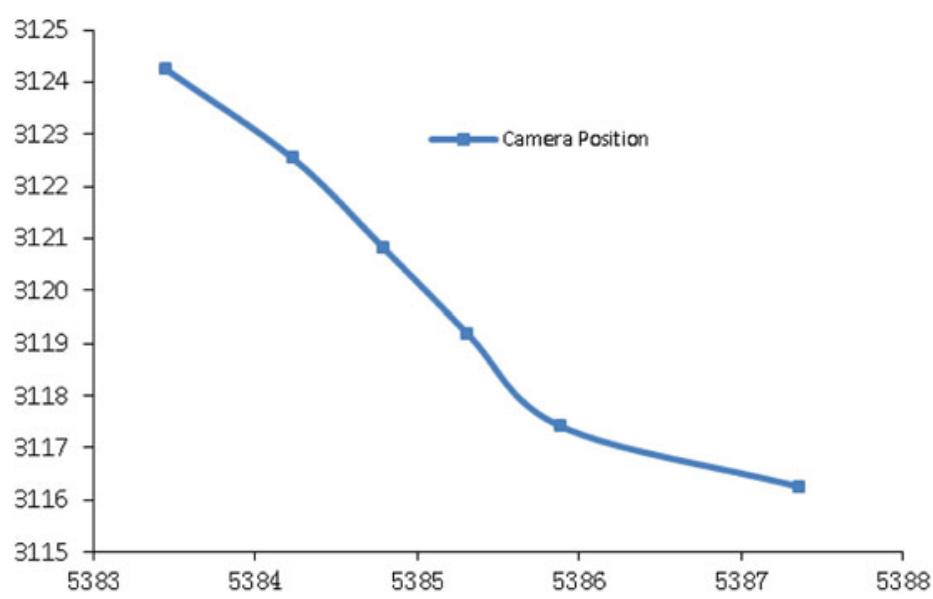

Figure 8. Exterior parameters of the image sequence.

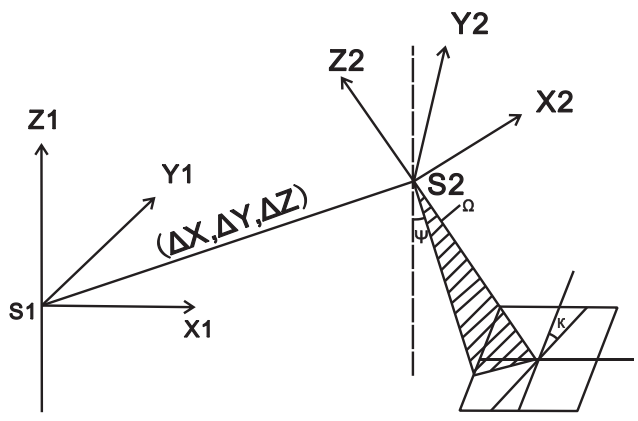

Figure 9. Spatial relationship between IMU and the camera.

projection centre and the geometry centre of the IMU; and (2) determining the rotation matrix between the image space coordinate system and the carrier coordinate system (original at the centre of the IMU).

The calibration is performed in an experiment ground with sufficient number of feature points. firstly, two points, named A and B, are selected, with their coordinates obtained via differential GPS, then Gauss projection is performed to obtain their Gaussian coordinates, so they can be used as control points in the following total station survey. The procedure is as follows:

- $\mathrm{n}$ feature points on the ground are selected and marked as control points in the resection process, and their $3 \mathrm{D}$ coordinates are obtained via total station survey. 
Images are shot with the camera and their exterior parameters are retrieved according to the resection theory, so the transformation matrix $R_{C}^{e}$ from image space coordinate system to Earth-Centred Earth-Fixed (ECEF) coordinate system is obtained.

- The carrier vehicle is placed in a suitable position and angle and kept stationary. The IMU continuously obtains measurement data. For a high accuracy IMU, the attitude (pitch, roll, yaw) of the carrier vehicle can be obtained through initial alignment, however, for a low accuracy IMU, in which the gyro is not able to sense the earth's rotation, we can only obtain pitch and roll angle; thus, the yaw angle should be obtained by other means. For our carrier vehicle, the base is designed in the shape of rectangle, and the IMU is mounted aligned to its centre line. So the coordinates of the four corner points of the carrier base are measured, and the carrier heading angle is obtained.

While the IMU stays stationary, it outputs the gravitational acceleration $g$ and the component of Earth's rotation angular velocity in the carrier coordinate system. However, an IMU with low accuracy cannot sense the Earth's rotation. Therefore, only the pitch and roll angle can be calculated from the IMU output.

The output gravitational acceleration while stationary is assumed as $g^{\mathrm{b}}$; that is:

$$
g^{b}=\left(\begin{array}{lll}
g_{x}^{b} & g_{y}^{b} & g_{z}^{b}
\end{array}\right)^{T}
$$

The component of the gravitational acceleration $\mathrm{g}$ in the local coordinate is assumed as $\mathrm{g}^{\mathrm{L}}$. This component can be easily retrieved as follows:

$$
\mathrm{g}^{\mathrm{L}}=\left[\begin{array}{lll}
0 & 0 & -\mathrm{g}
\end{array}\right]^{\mathrm{T}}
$$

These assumptions satisfy the following equation:

$$
\mathrm{g}^{\mathrm{b}}=\mathrm{R}_{\mathrm{L}}^{\mathrm{b}} \times \mathrm{g}^{\mathrm{L}}
$$

where $R_{L}^{b}$ is the rotation matrix between $g^{b}$ and $g^{L}$.

In detail, the above equation can be expressed as:

$$
\left\{\begin{array}{l}
g_{x}^{b}=-\sin r \times \cos p \times g \\
g_{y}^{b}=\sin p \times g \\
g_{z}^{b}=\cos r \times \cos p \times g
\end{array}\right.
$$

Thus, the expression for the calculation of the pitch and roll angles is:

$$
\left\{\begin{array}{l}
p=\sin ^{-1}\left(\frac{\mathrm{g}_{\mathrm{y}}^{\mathrm{b}}}{\mathrm{g}}\right) \\
r=-\tan ^{-1}\left(\frac{\mathrm{g}_{\mathrm{x}}^{\mathrm{b}}}{\mathrm{g}_{\mathrm{z}}^{\mathrm{b}}}\right)
\end{array}\right.
$$

The azimuth of the carrier can be presented by the midpoints of the front and back ends. The midpoint of the front end is marked as $C_{0}$, and that of the back end as $D_{0}$. 
Thus, the following equation is obtained:

$$
\begin{cases}C_{o X}=\frac{C_{L X}+C_{R X}}{2}, & D_{o X}=\frac{D_{L X}+D_{R X}}{2} \\ C_{o Y}=\frac{C_{L Y}+C_{R Y}}{2}, & D_{o Y}=\frac{D_{L Y}+D_{R Y}}{2} \\ C_{o H}=\frac{C_{L H}+C_{R H}}{2}, & D_{o H}=\frac{D_{L H}+D_{R H}}{2}\end{cases}
$$

The following equation is used to retrieve the carrier azimuth $a$ :

$$
a=\arctan \left[\frac{C_{o Y}-D_{o Y}}{C_{o X}-D_{o X}}\right]
$$

Hence, the attitude matrix of the carrier can be directly calculated using the $p, r$, and $y$ values:

$$
R_{b}^{L}=\left(\begin{array}{ccc}
\cos r \cos y-\sin r \sin y \sin p & -\sin y \cos p & \sin r \cos y+\cos r \sin y \sin p \\
\cos r \sin y+\sin r \cos y \sin p & \cos y \cos p & \sin r \sin y-\cos r \cos y \sin p \\
-\sin r \cos p & \sin p & \cos r \cos p
\end{array}\right)
$$

where $y=2 \pi-a$.

When the $R_{b}^{L}$ and $R_{C}^{e}$ values are known, the rotation matrix can be easily calculated:

$$
R_{C}^{b}=\left(R_{b}^{L}\right)^{T} \cdot R_{e}^{L} \cdot R_{C}^{e}
$$

where $R_{e}^{L}$ is the rotation matrix from the ECEF to the local system, which depends on the latitude and longitude of the carrier.

$R_{e}^{L}$ can be expressed as follows:

$$
R_{e}^{L}=\left(\begin{array}{ccc}
-\sin \lambda & \cos \lambda & 0 \\
-\sin \varphi \cos \lambda & -\sin \varphi \sin \lambda & \cos \varphi \\
\cos \varphi \cos \lambda & \cos \varphi \sin \lambda & \sin \varphi
\end{array}\right)
$$

where:

$\varphi$ is latitude.

$\lambda$ is longitude.

$\varphi$ and $\lambda$ are obtained from the linear elements of camera's exterior parameters.

$R_{C}^{e}$ is the rotation matrix from image space coordinate system to ECEF.

$R_{C}^{e}$ can be expressed as follows:

$R_{C}^{e}=\left(\begin{array}{ccc}\cos \phi \cos \kappa-\sin \phi \sin \omega \sin \kappa & -\cos \phi \sin \kappa-\sin \phi \sin \omega \cos \kappa & -\sin \phi \cos \omega \\ \cos \omega \sin \kappa & \cos \omega \cos \kappa & -\sin \omega \\ \sin \phi \cos \kappa+\cos \phi \sin \omega \sin \kappa & -\sin \phi \sin \kappa+\cos \phi \sin \omega \cos \kappa & \cos \phi \cos \omega\end{array}\right)$

where, $\phi, \omega, \kappa$ are three Euler angles from image space coordinate system to ECEF, which are angle elements of the camera's exterior parameters.

The coordinates can be retrieved from the centre of the IMU, and its offset to the centre of the camera can be obtained. 
4.3. Optimization with the Integrated IMU. As a key part of the algorithm, the integration of the VO and the IMU using an EKF will be implemented; actually, a wheel odometry is also used in our system which can provide velocity information of the carrier. Two approaches are considered: one is that, when the image features are sufficiently rich, we use the estimated position and attitude of the camera via bundle adjustment to calibrate the gyro drifts, accelerometer bias, scale factor error of the wheel odometry with EKF; the residuals of the camera position and attitude can also be estimated. Once all these sensor errors are calibrated, a high accuracy can be obtained and used to support the estimation of the parameters for situations in which the image features are too poor to determine the camera's position or attitude; this needs another approach to be considered. Under this situation, only IMU measurements and wheel odometry data are used for integration, and the estimated position and attitude are transformed and assigned to the camera.

VO and IMU are fused via loose coupling, in which each subsystem is taken as an independent estimator. In the current paper, an indirect Kalman filter is used to estimate the system errors. The error state considers the INS navigation parameter errors, IMU errors, and scale factor errors for the wheel odometry. The dynamic equation of the system is based on the INS error equation.

The error state vector can be written as

$$
X=\left(\delta r^{L}, \delta v^{L}, \delta \varepsilon^{L}, d, b, \delta k\right)
$$

where $\delta r^{L}, \delta v^{L}, \delta \varepsilon^{L}, d$ and $b$ are 3D position errors, 3D velocity errors, 3D misalignment angles, 3D gyro drifts, and 3D accelerometer bias for the INS, respectively.

$\delta k$ is the scale factor error of the wheel odometry.

The error state vector can also be written as

$$
X=\left[\begin{array}{l}
X_{\text {ins }} \\
X_{o d}
\end{array}\right]
$$

The system dynamic equation is described as follows:

$$
\dot{X}=\left[\begin{array}{cc}
F_{\text {ins }} & O \\
15 \times 15 & 15 \times 1 \\
O & 0
\end{array}\right]\left[\begin{array}{c}
X_{\text {ins }} \\
X_{\text {od }}
\end{array}\right]+G \cdot W
$$

where $F_{\text {ins }}$ is the coefficient matrix of the INS error equation, which can be derived from the INS navigation equation in the local-level frame, and $G$ is the process noise dynamic matrix.

The inputs to the Kalman filter include the velocities from the INS outputs and wheel odometry measurements, the coordinates from the INS outputs and VO outputs, and the attitude angles from the INS outputs and VO outputs. The measurement equation can be written as follows:

$$
\left(\begin{array}{c}
Z_{v} \\
Z_{r} \\
Z_{a}
\end{array}\right)=\left(\begin{array}{c}
H_{v} \\
H_{r} \\
H_{a}
\end{array}\right) \cdot X+\left(\begin{array}{c}
V_{v} \\
V_{r} \\
V_{a}
\end{array}\right)
$$

where:

$Z_{v}, Z_{r}, Z_{a}$ are filter measurements.

$H_{v}, H_{r}, H_{a}$ are matrix measurements.

$V_{v}, V_{r}, V_{a}$ are the residuals. 
The velocity errors can be denoted as:

$$
Z_{v}=V_{i n s}^{n}-R_{b}^{n} V_{o d}^{b}
$$

where $V_{o d}^{b}$ is the velocity of the vehicle in a forward direction as measured using the wheel odometry.

The measurement matrix for the velocity errors can be derived as follows:

$$
H_{v}=\left(\begin{array}{cccccccccccccccc}
0 & 0 & 0 & 1 & 0 & 0 & 0 & -v_{o d}^{u} & v_{o d}^{n} & 0 & 0 & 0 & 0 & 0 & 0 & -v_{o d}^{e} \\
0 & 0 & 0 & 0 & 1 & 0 & v_{o d}^{u} & 0 & -v_{o d}^{e} & 0 & 0 & 0 & 0 & 0 & 0 & -v_{o d}^{n} \\
0 & 0 & 0 & 0 & 0 & 1 & -v_{o d}^{n} & v_{o d}^{e} & 0 & 0 & 0 & 0 & 0 & 0 & 0 & -v_{o d}^{u}
\end{array}\right)
$$

The position errors can be denoted as:

$$
Z_{r}=r_{\text {ins }}-r_{\text {image }}
$$

where:

$r_{\text {ins }}$ represents the position of the vehicle obtained from IMU.

$r_{\text {image }}$ represents the position of the vehicle obtained from VO.

The position vector $r_{\text {image }}$ should be expressed using the origin in the IMU centre by subtracting the lever arm between IMU and the camera.

The measurement matrix for the position errors can be easily written as follows:

$$
H_{r}=\left(\begin{array}{llllllllllllllll}
1 & 0 & 0 & 0 & 0 & 0 & 0 & 0 & 0 & 0 & 0 & 0 & 0 & 0 & 0 & 0 \\
0 & 1 & 0 & 0 & 0 & 0 & 0 & 0 & 0 & 0 & 0 & 0 & 0 & 0 & 0 & 0 \\
0 & 0 & 1 & 0 & 0 & 0 & 0 & 0 & 0 & 0 & 0 & 0 & 0 & 0 & 0 & 0
\end{array}\right)
$$

The attitude errors can be denoted as:

$$
Z_{a}=a_{\text {ins }}-a_{\text {image }}
$$

where:

$a_{i n s}$ represents the attitude of the vehicle obtained from IMU.

$a_{\text {image }}$ represents the attitude of the vehicle obtained from VO.

The attitude vector $a_{\text {image }}$ should be denoted as the attitude that relates the body frame with the local-level frame, by taking into account the calibrated result of the transformation matrix between IMU and the camera.

The measurement matrix for the attitude errors can be used to determine the relationship between the attitude errors and the misalignment angles. The equation can thus be derived as follows:

$$
H_{a}=\left(\begin{array}{cccccccccccccccc}
0 & 0 & 0 & 0 & 0 & 0 & \cos y & \sin y & 0 & 0 & 0 & 0 & 0 & 0 & 0 & 0 \\
0 & 0 & 0 & 0 & 0 & 0 & -\frac{\sin y}{\cos p} & \frac{\cos y}{\cos p} & 0 & 0 & 0 & 0 & 0 & 0 & 0 & 0 \\
0 & 0 & 0 & 0 & 0 & 0 & \frac{\sin y \sin p}{\cos p} & -\frac{\cos y \sin p}{\cos p} & 1 & 0 & 0 & 0 & 0 & 0 & 0 & 0
\end{array}\right)
$$




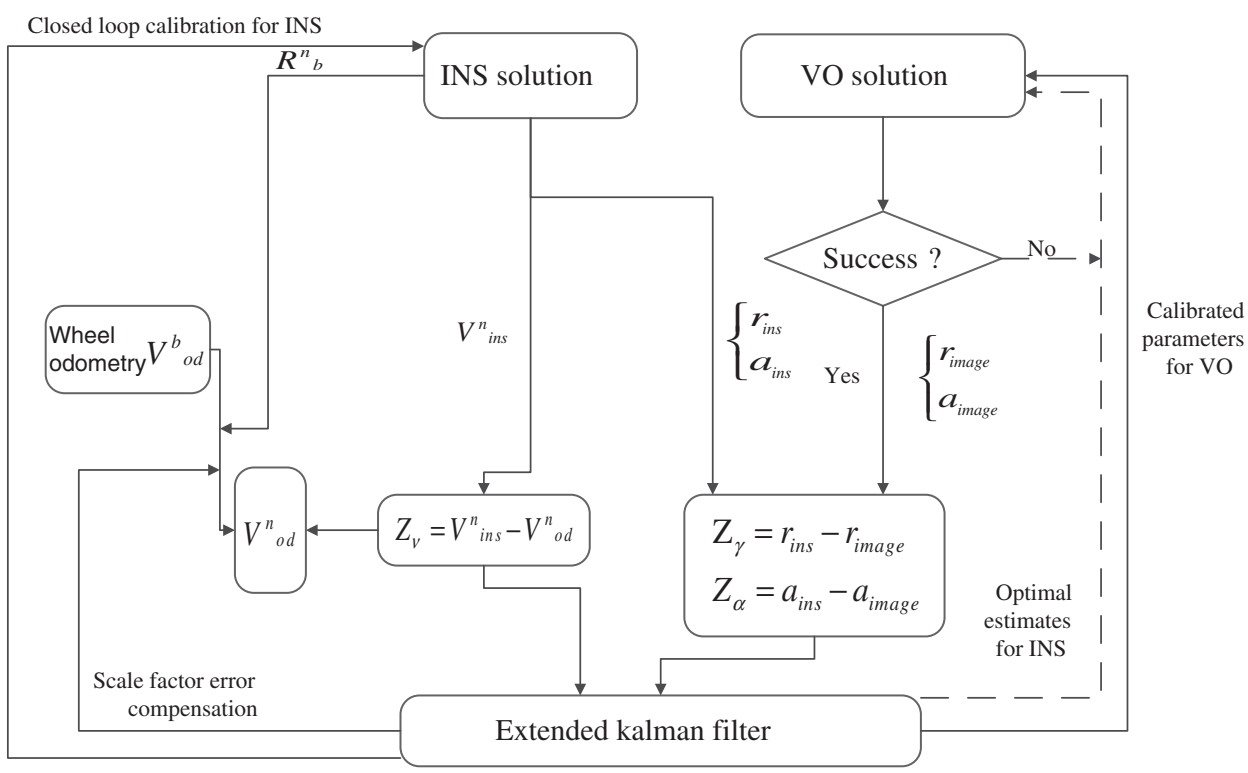

Figure 10. Visual odometry/IMU integration Kalman filter.

In a filter circle, the state error is estimated and compensated in the system, and the parameters for $\mathrm{VO}$ are also adjusted

$$
\begin{aligned}
& \hat{r}_{\text {image }}=r_{\text {ins }}-H_{r} \cdot \hat{X} \\
& \hat{a}_{\text {image }}=a_{\text {ins }}-H_{a} \cdot \hat{X}
\end{aligned}
$$

where $\hat{X}$ is the output of the Kalman filter for the error state estimation, and $\hat{r}_{\text {image }}, \hat{a}_{\text {image }}$ are the adjustment parameters for VO.

Figure 10 summarizes the Kalman filter frame for the visual odometry/IMU integration. The system is a closed loop Kalman filter, the outputs of which are fed back to the system. When the solution for VO succeeds, the calibrated parameters for VO after the filtration process return, and the estimated IMU errors and wheel odometry error are calibrated. Thus, the integrated system can maintain high accuracy for some time, even under the occasional short-lived VO solution failures. When the VO solution fails, the only inputs are the velocities from INS and the wheel odometry, with no VO inputs. Kalman filtering for the integrated IMU/wheel odometry system is implemented, and the outputs, which are the optimal estimated INS parameters (position and attitude), are passed on to the camera. In both cases, the coordinates of the matched feature points would be recalculated via a forward intersection using the estimated parameters for Kalman filtering for the camera.

5. EXPERIMENT AND RESULTS. The author developed a hardware system, including a stereo camera pair, an IMU, a wheel sensor, a computer, and a router, all mounted on a navigation vehicle, as well as a computational software based on the algorithms presented in the current paper. The IMU used in our system consists of three Fibre Optic Gyros and three MEMS Accelerometers. Gyro bias stability is 


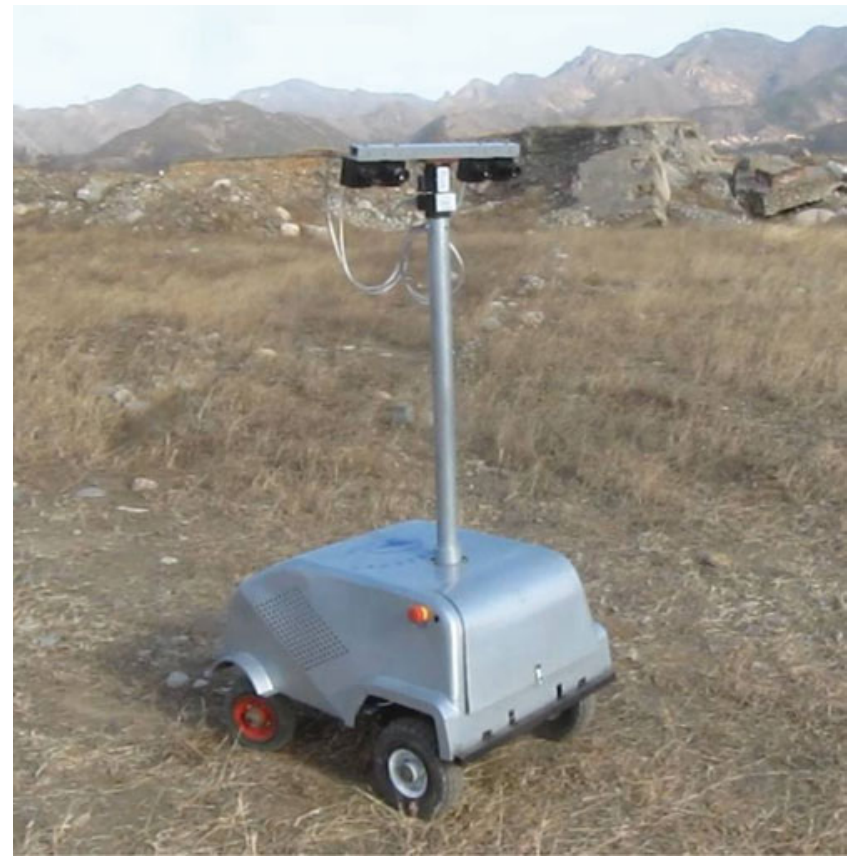

Figure 11. Navigation platform in an experiment.

$0.005 \mathrm{deg} / \mathrm{s}$. Accelerometer bias stability is $10 \mathrm{mg}$. The type of stereo cameras is progressive scanning CCD, the baseline is $30 \mathrm{~cm}$, the focal length is $12 \mathrm{~mm}$, and the image frame is $696 \times 520$ pixels, with pixel size of $6 \cdot 45 \mu \mathrm{m} \times 6 \cdot 45 \mu \mathrm{m}$, the field of view in horizontal and vertical are $42^{\circ}$ and $31^{\circ}$. The system is capable of data collection and navigation solution in real time.

We conducted an experiment in a realistic situation to demonstrate the validity of our algorithm. The experiment is performed in an open square located in Beijing, see Figure 11. The entire process lasts for approximately 45 minutes, with the vehicle running at a distance of about $1150 \mathrm{~m}$. The data set collected includes images, IMU data, wheel sensor data, and RTK GPS data. In the data collection process, the images are captured at a rate of $2 \mathrm{~Hz}$, and the total images collected are over 4300 frames. The wheel sensor output velocity of the vehicle is $2 \mathrm{~Hz}$, and the IMU data rate is $200 \mathrm{~Hz}$. The RTK GPS outputs real-time differential results at $1 \mathrm{~Hz}$ with an accuracy of several centimetres, and is used as a position truth to evaluate the accuracy of the test results. The surroundings of the vehicle path are very complex, with a considerable amount of grass and scattered stones on the ground for most sections of the route. Some sections were bare ground.

Three different methods, namely, visual odometry, IMU/WO integration, VO/IMU integration, are used for data processing using the collected data sets; a comparison of the results is also conducted. Occasional failures occur when the VO algorithm is used to compute all the image frames of the data sets. The analysis shows that solution failure occurs when the number of feature points in the image is too low, such as in highly reflective bare ground. This failure also occurs when the feature matching is difficult to perform, such as in a monotonous grass background. To fill these gaps, the IMU/wheel odometry integration is used to link the failed image frames. 


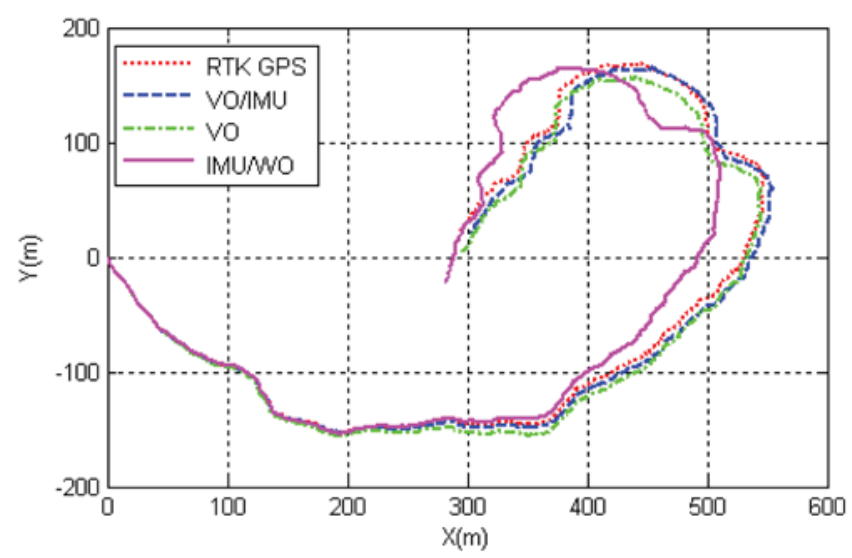

Figure 12. Trajectories obtained from the different solutions.

Figure 12 shows the trajectories of the vehicle in the experiment; the four trajectories obtained from RTK GPS, IMU/WO integration, VO solution, and $\mathrm{VO} / \mathrm{IMU}$ integration are also shown. The trajectory from the VO/IMU integration is very close to that of RTK GPS. The deviations between the RTK GPS trajectory and the three others are also found to increase with the running distance. Table 5 lists the navigation errors of the three methods. The position error of the terminal point of the VO/IMU integration is much smaller than that of the IMU/wheel odometry integration and the VO solution, with running distance of $1150 \mathrm{~m}$, an accumulated error of $8.6 \mathrm{~m}$, and a relative error percentage of $0.75 \%$. During the experiment, there are six times of short-time visual odometry failure, but we can see that the accuracy of the integrated system is not seriously affected by the visual odometry failure.

The innovation of Kalman filtering is illustrated in Figure 13 and Figure 14, including position innovation and attitude innovation. It can be seen from Figure 13 that the maximum position innovation in $\mathrm{X}, \mathrm{Y}, \mathrm{Z}$ directions is less than $0.15 \mathrm{~m}$, and in most cases less than $0.05 \mathrm{~m}$. It can be seen from Figure 14 that the maximum attitude innovation in pitch, roll, yaw angles is less than $0 \cdot 1^{\circ}$, and in most cases less than $0.02^{\circ}$. The innovation indicates that the Kalman filtering process is relatively stable, the visual odometry and IMU integrated algorithm in this paper is valid and efficient.

6. CONCLUSIONS. The current article introduces in detail the integrated vision navigation system matching concept, research background, and recent developments both domestic and abroad. The proposed passage applies a reliable estimation method that improves the matching results of the SURF method, significantly reducing the rate of wrong matches while avoiding extra calculation time. The proposed system makes use of the improved results from the SURF method as well as the relevant theories on photogrammetry. Moreover, the proposed system realizes the measurement of $3 \mathrm{D}$ coordinates and an image sequence-based fast coordinate extrapolation. At the same time, relevant programs are developed and applied, and experimental results are collected and analysed. Finally, the proposed system uses the integrated IMU data to further improve the accumulated error of the model, which would be the foundation for further studies. 
Table 5. Solution errors compared to GPS.

\begin{tabular}{lcc}
\hline Solution & $\begin{array}{c}\text { Error in position } \\
(\mathrm{m})\end{array}$ & $\begin{array}{c}\text { Error percentage } \\
\text { of the distance }\end{array}$ \\
\hline IMU/WO & $52 \cdot 3$ & $4 \cdot 5 \%$ \\
VO & $18 \cdot 7$ & $1.6 \%$ \\
VO/IMU & 8.6 & $0 \cdot 75 \%$ \\
\hline
\end{tabular}

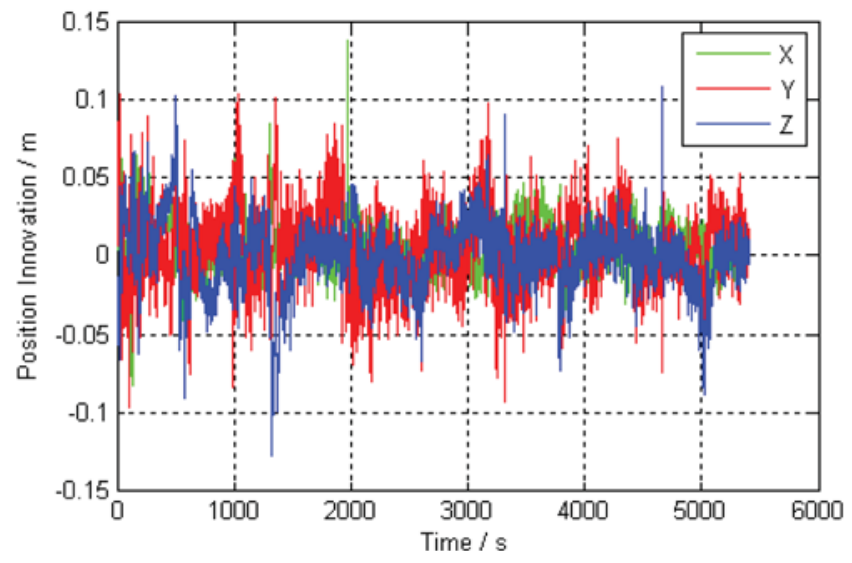

Figure 13. Position innovation of Kalman filtering.

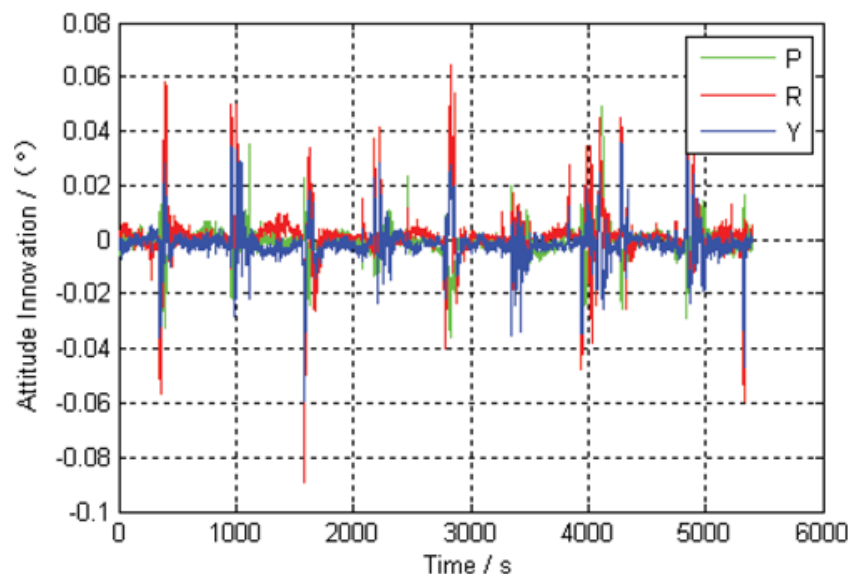

Figure 14. Attitude innovation of Kalman filtering.

The SURF method can execute a faster common point matching and perform realtime matching. However, several wrong matching points are still obtained, affecting the application range for the matching results. The improved SURF method not only rapidly matches the common points, it also avoids many wrong matching points, contributing to the overall accuracy. However, the improved method reduces the amount of matching points. Based on the simple and efficient $3 \mathrm{D}$ coordinate 
measurement method of the basic theory of photogrammetry, the coordinates of the image point in object space can easily be obtained. However, a number of technical problems on the measurement of the image sequence-based coordinates remain to be resolved. The IMU can provide an exterior orientation for the image, which greatly simplifies the calculation. The combined treatment of IMU data will be investigated in the future.

\section{ACKNOWLEDGEMENT}

The work in this paper is supported by National Basic Research Program of China (Project No. 2012CB957702).

\section{REFERENCES}

Bay, H., Tuytelaars, T. and Van Gool, L. (2006). SURF: Speeded-Up Robust Features. Proceedings of ECCV 2006, 3951, 404-417.

Bayoud, F. and Skaloud, J. (2008). Vision-Aided Inertial Navigation System For Robotic Mobile Mapping. Journal of Applied Geodesy, 2, 39-52.

Castro, E. De and Morandi, C. (1987). Registration of Translated and Rotated Images Using Finite Fourier Transforms. IEEE Transactions on Pattern Analysis and Machine Intelligence, 9(5), 700-703.

Cheng, Y., Maimone, M. and Matthies, L. (2005). Visual Odometry on the Mars Exploration Rovers. Proceedings of the IEEE International Conference on Systems, Man and Cybernetics, 1, 903-910.

DeSouza, G. N. and Kak, A. C. (2002). Vision for Mobile Robot Navigation: A Survey. IEEE Transaction on Pattern Analysis and Machine Intelligence, 24(2), 237-267.

Konolige, K., Agrawal, M. and Solà, J. (2007). Large Scale Visual Odometry for Rough Terrain. Proceedings of the International Symposium on Robotics Research, 2, 201-212.

Lucas, B. D. and Kanade, T. (1981). An Iterative Image Registration Technique with an Application to Stereo Vision. Proceedings of IJCAI1981, 674-679.

Maimone, M., Cheng, Y. and Matthies, L. (2007). Two Years of Visual Odometry on the Mars Exploration Rovers. Journal of Field Robotics, 24(2), 169-186.

Mikolajczyk, K. and Schmid, C. (2005). A Performance Evaluation of Local Descriptors. IEEE Transactions on Pattern Analysis and Machine Intelligence, 27, 1615-1630.

Pei, C. (2010). The Research for the Binocular Stereo Matching Based on the Computer Vision. Master Dissertation In Jiangsu University, China (in Chinese).

Song, C. B. (2004). Study on Image Matching in the Field of Stereo Vision Based on Line Feature. Master Dissertation In Wuhan University, China (in Chinese).

Su, L. C. and Zhu, F. (2005). Design of a Novel Stereo Vision Navigation System for Mobile Robots. Proceedings of IEEE International Conference on Robotics and Biomimetics (ROBIO'05), pp. 611-614.

Thorpe, C., Hebert, M.H., Kanade, T. and Shafer, S. A. (1988). Vision and Navigation for the Carnegie-Mellon Navlab. IEEE Transaction on Pattern Analysis and Machine Intelligence, 10(3), $362-373$. 